\title{
Theoretical and Numerical Aspects of a Third-order Three-point Nonhomogeneous Boundary Value Problem
}

\author{
A. L. M. MARTINEZ ${ }^{1 *}$, M. R. A. FERREIRA ${ }^{2}$ and E. V. CASTELANI ${ }^{3}$ \\ Received on December 03, 2018 / Accepted on February 21, 2019
}

\begin{abstract}
In this paper we are considering a third-order three-point equation with nonhomogeneous conditions in the boundary. Using Krasnoselskii's Theorem and Leray-Schauder Alternative we provide existence results of positive solutions for this problem. Nontrivials examples are given and a numerical method is introduced.
\end{abstract}

Keywords: numerical solutions, third-order, boundary value problem, Krasnoselskii's Theorem.

\section{INTRODUCTION}

Multi-point boundary value problems there has been attention of several studies mainly focused on the existence of solutions with qualitative and quantitative aspects, we recommend [2, 3, 4, 5, $6,7,8,9,10,11,12,14,15]$ and the references therein. It is well known that the Krasnoselskii's fixed point theorem, Avery-Peterson and Leggett-Williams theorems are massively used in this line.

In this paper, motived by [13], we discuss the existence of a positive solution for the third-order boundary value problem:

$$
\begin{gathered}
u^{\prime \prime \prime}+f\left(t, u, u^{\prime}\right)=0, \quad 0<t<1, \\
u(0)=u^{\prime}(0)=0, \quad u^{\prime}(1)-\alpha u^{\prime}(\eta)=\lambda,
\end{gathered}
$$

where $\eta \in(0,1), \alpha \in\left[0, \frac{1}{\eta}\right)$ are constants and $\lambda \in(0, \infty)$ is a parameter. Essentially, we combine Leray-Schauder Alternative and Krasnoselskii's theorem to show the existence of a positive solution for (1.1)-(1.2) without supposing superlinearity on $f$. Numerical solutions are poorly

\footnotetext{
*Corresponding author: André L. M. Martinez - E-mail: andrelmmartinez@yahoo.com.br - https://orcid.org/00000003-1888-648X

${ }^{1}$ Departamento Acadêmico de Matemática, Universidade Tecnológica Federal do Paraná, Cornélio Procópio, Paraná, Brazil - E-mail: andrelmmartinez@yahoo.com.br

${ }^{2}$ Colegiado de Matemática, Universidade Estadual do Norte do Paraná, Cornélio Procópio, Paraná, Brazil - E-mail: marceloraferreira@gmail.com

3 Departamento de Matemática, Universidade Estadual de Maringá, Maringá, Paraná, Brazil - E-mail: evcastelani@uem.br
} 
explored, thus we complement this work presenting a numerical study for (1.1)-(1.2) based on Banach's Contraction Principle.

\section{BACKGROUND MATERIAL}

We begin this section by stating the following results.

Theorem 1. Let $E$ be a Banach space, $C \subset E$ a closed and convex set, $\Omega$ an open set in $C$ and $p \in \Omega$. Then each completely continuous mapping $T: \bar{\Omega} \rightarrow C$ has at least one of the following properties:

(A1) Thas a fixed point in $\bar{\Omega}$.

(A2) There are $u \in \partial \Omega$ and $\lambda \in(0,1)$ such that $u=\lambda T(u)+(1-\lambda) p$.

Theorem 2. Let $E$ be a Banach space and let $K \subset E$ be a cone in E. Assume $\Omega_{1}, \Omega_{2}$ are bounded open subsets of $E$ with $0 \in \Omega_{1}, \bar{\Omega}_{1} \subset \Omega_{2}$, and let $T: K \cap\left(\bar{\Omega}_{2} \backslash \Omega_{1}\right) \rightarrow K$ be a completely continuous operator such that, either

(B1) $\|T u\| \leq\|u\|, u \in K \cap \partial \Omega_{1}$, and $\|T u\| \geq\|u\|, u \in K \cap \partial \Omega_{2}$, or

(B2) $\|T u\| \geq\|u\|, u \in K \cap \partial \Omega_{1}$, and $\|T u\| \leq\|u\|, u \in K \cap \partial \Omega_{2}$.

Then $T$ has a fixed point in $K \cap\left(\bar{\Omega}_{1} \backslash \Omega_{1}\right)$.

The first theorem is a well-known Leray-Schauder alternative and the second theorem is due to Krasnoselskii, see [1].

Let us set an auxiliary problem that will be useful in our context.

$$
\begin{gathered}
u^{\prime \prime \prime}+f\left(t, x, x^{\prime}\right)=0, \quad 0<t<1, \\
u(0)=u^{\prime}(0)=0, \quad u^{\prime}(1)-\alpha u^{\prime}(\eta)=\lambda .
\end{gathered}
$$

Related to this problem we have an important lemma.

Lemma 3. Let $x \in C^{1}[0,1]:=\left\{x \in C^{1}[0,1], t \in[0,1]\right\}$, then we have a unique solution for (2.1)(2.2). Moreover, this solution is expressed by

$$
\begin{array}{r}
u(t)=\int_{0}^{1} G(t, s) f\left(s, x(s), x^{\prime}(s)\right) d s+\frac{\alpha t^{2}}{2(1-\alpha \eta)} \int_{0}^{1} G_{1}(\eta, s) f\left(s, x(s), x^{\prime}(s)\right) d s+ \\
+\frac{\lambda t^{2}}{2(1-\alpha \eta)},
\end{array}
$$

where $G$ is the Green's function:

$$
G(t, s)=\frac{1}{2}\left\{\begin{array}{lr}
\left(2 t-t^{2}-s\right) s, & s \leq t \\
(1-s) t^{2}, & t \leq s
\end{array}\right.
$$


and

$$
G_{1}(t, s)=\frac{\partial G(t, s)}{\partial t}=\left\{\begin{array}{ll}
(1-t) s, & s \leq t \\
(1-s) t, & t \leq s
\end{array} .\right.
$$

Proof. If $u(t)$ is solution of (2.1), we can suppose that

$$
u(t)=-\frac{1}{2} \int_{0}^{t}(t-s)^{2} f\left(s, x, x^{\prime}\right) d s+A t^{2}+B t+C .
$$

From condition (2.2), we have $B=C=0$ and

$$
A=\frac{1}{2(1-\alpha \eta)} \int_{0}^{1}(1-s) f\left(s, x, x^{\prime}\right) d s-\frac{\alpha}{2(1-\alpha \eta)} \int_{0}^{\eta}(\eta-s) f\left(s, x, x^{\prime}\right) d s+\frac{\lambda}{(1-\alpha \eta)}
$$

Thus (2.1)-(2.2) has a unique solution. Furthermore $u(t)=-\frac{1}{2} \int_{0}^{t}(t-s)^{2} f\left(s, x, x^{\prime}\right) d s+$

$$
\begin{aligned}
& \frac{t^{2}}{2(1-\alpha \eta)} \int_{0}^{1}(1-s) f\left(s, x, x^{\prime}\right) d s \\
& \quad-\frac{\alpha t^{2}}{2(1-\alpha \eta)} \int_{0}^{\eta}(\eta-s) f\left(s, x, x^{\prime}\right) d s+\frac{\lambda t^{2}}{2(1-\alpha \eta)} \\
&=-\frac{1}{2} \int_{0}^{t}(t-s)^{2} f\left(s, x, x^{\prime}\right) d s+\frac{t^{2}}{2} \int_{0}^{1}(1-s) f\left(s, x, x^{\prime}\right) d s \\
& \quad+\frac{\alpha \eta t^{2}}{2(1-\alpha \eta)} \int_{0}^{1}(1-s) f\left(s, x, x^{\prime}\right) d s-\frac{\alpha t^{2}}{2(1-\alpha \eta)} \int_{0}^{\eta}(\eta-s) f\left(s, x, x^{\prime}\right) d s+\frac{\lambda t^{2}}{2(1-\alpha \eta)} \\
&=\frac{1}{2} \int_{0}^{t}\left(-t^{2}+2 s t-s^{2}\right) f\left(s, x, x^{\prime}\right) d s+\frac{1}{2} \int_{0}^{t}(1-s) t^{2} f\left(s, x, x^{\prime}\right) d s \\
& \quad+\frac{1}{2} \int_{t}^{1}(1-s) t^{2} f\left(s, x, x^{\prime}\right) d s+\frac{\alpha t^{2}}{2(1-\alpha \eta)} \int_{0}^{\eta}(1-s) \eta f\left(s, x, x^{\prime}\right) d s \\
& \quad+\frac{\alpha t^{2}}{2(1-\alpha \eta)} \int_{\eta}^{1}(1-s) \eta f\left(s, x, x^{\prime}\right) d s-\frac{\alpha t^{2}}{2(1-\alpha \eta)} \int_{0}^{\eta}(\eta-s) f\left(s, x, x^{\prime}\right) d s+\frac{\lambda t^{2}}{2(1-\alpha \eta)} \\
&=\frac{1}{2} \int_{0}^{t}\left(2 t-t^{2}-s\right) s f\left(s, x, x^{\prime}\right) d s+\frac{1}{2} \int_{t}^{1}(1-s) t^{2} f\left(s, x, x^{\prime}\right) d s \\
& \quad+\frac{\alpha t^{2}}{2(1-\alpha \eta)}\left(\int_{0}^{\eta}(1-\eta) s f\left(s, x, x^{\prime}\right) d s+\int_{\eta}^{1} \eta(1-s) f\left(s, x, x^{\prime}\right) d s\right)+\frac{\lambda t^{2}}{2(1-\alpha \eta)} \\
&= \int_{0}^{1} G(t, s) f\left(s, x, x^{\prime}\right) d s+\frac{\alpha t^{2}}{2(1-\alpha \eta)} \int_{0}^{1} G_{1}(\eta, s) f\left(s, x, x^{\prime}\right) d s+\frac{\lambda t^{2}}{2(1-\alpha \eta)} .
\end{aligned}
$$

Defining $x(t)=u(t)$ in Lemma 3 is easy to see that the solution of (1.1)-(1.2) can be expressed as fixed point of the operator $T: C^{1}[0,1] \rightarrow C^{1}[0,1]$ defined by:

$$
T u(t)=\int_{0}^{1} G(t, s) f\left(s, u, u^{\prime}\right) d s+\frac{\alpha t^{2}}{2(1-\alpha \eta)} \int_{0}^{1} G_{1}(\eta, s) f\left(s, u, u^{\prime}\right) d s+\frac{\lambda t^{2}}{2(1-\alpha \eta)}
$$


Remark 4. Related to $G$ and $G_{1}$ we have useful properties that will be used in the next section.

- For all $(t, s) \in[0,1] \times[0,1]$ :

$$
0 \leq G_{1}(t, s) \leq(1-s) s
$$

- For all $(t, s) \in[0,1] \times[0,1]$ :

$$
G(t, s) \leq G_{1}(1, s)=\frac{1}{2}(1-s) s
$$

\section{POSITIVE SOLUTIONS}

Let $E=\left\{u \in C^{1}[0,1]: u(0)=0\right\}$, where $C^{1}[0,1]$ be the Banach space of continuously differentiable functions in $[0,1]$ equipped with

$$
\|u\|_{E}=\max \left\{\|u\|_{\infty},\left\|u^{\prime}\right\|_{\infty}\right\} .
$$

Remark 1. If $u \in E$ then $T u$ satisfies $T u(0)=0$. Besides $\left\|(T u)^{\prime}\right\|_{\infty} \geq\|T u\|_{E}$.

In order to prove the existence we need to consider some basic assumptions.

(H1) There exist positive constants $A, B$ and $\beta$ such that

$\max _{\left(s, v_{1}, v_{2}\right) \in[0,1] \times[-\beta, \beta] \times[-\beta, \beta]}\left\{\left|f\left(s, v_{1}, v_{2}\right)\right|\right\} \leq \frac{\beta(1-\alpha \eta) 6 B}{1+\alpha(1-\eta)}$

- $\lambda \leq A \beta(1-\alpha \eta)$

- $A+B \leq 1$

Lemma 2. Suppose that (H1) holds. Thus the problem (1.1)-(1.2) has a solution $u^{*} \in E$ with $\left\|u^{*}\right\|_{E} \leq \beta$.

Proof. Let us consider the Theorem 1 with $p=0$ and $\Omega=\left\{u \in E:\|u\|_{E}<\beta\right\}$.

We claim that $T$ is continuous and completely continuous. In fact, the continuity follows immediately from the Lebesgue dominated convergence theorem and noting that

$$
\begin{aligned}
\left|T(u)(t)-T\left(u_{n}\right)(t)\right| \leq & \int_{0}^{1} G(t, s)\left|f\left(s, u(s), u^{\prime}(s)\right)-f\left(s, u_{n}(s), u_{n}^{\prime}(s)\right)\right| d s+ \\
& +\left|\frac{\alpha t^{2}}{2(1-\alpha \eta)}\right| \int_{0}^{1} G_{1}(\eta, s)\left|f\left(s, u(s), u^{\prime}(s)\right)-f\left(s, u_{n}(s), u_{n}^{\prime}(s)\right)\right| d s \\
& \quad \leq \int_{0}^{1} G 1(1, s)\left|f\left(s, u(s), u^{\prime}(s)\right)-f\left(s, u_{n}(s), u_{n}^{\prime}(s)\right)\right| d s+
\end{aligned}
$$




$$
+\left|\frac{\alpha}{2(1-\alpha \eta)}\right| \int_{0}^{1} G_{1}(\eta, s)\left|f\left(s, u(s), u^{\prime}(s)\right)-f\left(s, u_{n}(s), u_{n}^{\prime}(s)\right)\right| d s,
$$

with $u_{n}, u \in E$. To show complete continuity we will use the Arzela-Ascoli's theorem. Let $\Omega \subseteq E$ be bounded, in other words, there exists $\Lambda_{0}>0$ with $\|u\| \leq \Lambda_{0}$ for each $u \in \Omega$. Now if $u \in \Omega$ we have

$$
\begin{aligned}
\left|(T u)^{\prime}(t)\right| & =\left|\int_{0}^{1} G_{1}(t, s) f\left(s, u, u^{\prime}\right) d s+\frac{\alpha t}{1-\alpha \eta} \int_{0}^{1} G_{1}(\eta, s) f\left(s, u, u^{\prime}\right)+\frac{\lambda t}{1-\alpha \eta}\right| \\
& \leq \int_{0}^{1}\left|G_{1}(t, s) f\left(s, u, u^{\prime}\right) d s\right|+\frac{\alpha t}{1-\alpha \eta} \int_{0}^{1}\left|G_{1}(\eta, s) f\left(s, u, u^{\prime}\right)\right|+\left|\frac{\lambda t}{1-\alpha \eta}\right| \\
& \leq \max _{t \in[0,1]} \frac{1-\alpha \eta+\alpha t}{1-\alpha \eta} \int_{0}^{1}\left|(1-s) s f\left(s, u, u^{\prime}\right)\right| d s+\left|\frac{\lambda t}{1-\alpha \eta}\right| \\
& \leq \frac{1+\alpha(-\eta+1)}{1-\alpha \eta} \int_{0}^{1}|(1-s) s|\left|f\left(s, u, u^{\prime}\right)\right| d s+\left|\frac{\lambda}{1-\alpha \eta}\right| .
\end{aligned}
$$

Then $T \Omega$ is a bounded equicontinuous family on $[0,1]$. Consequently the Arzela-Ascoli theorem implies $T: E \rightarrow E$ is completely continuous.

In addition, suppose there are $u \in \partial \Omega$ and $\lambda \in(0,1)$ with $u(x)=\lambda T u(x)$. According (H1) we have:

$$
\begin{aligned}
& \|T u\|_{E}<\left\|(T u)^{\prime}\right\|_{\infty}=\max _{t \in[0,1]}\left|(T u)^{\prime}(t)\right|, \\
& \leq \max _{t \in[0,1]} \frac{1+\alpha(-\eta+1)}{1-\alpha \eta} \int_{0}^{1}|(1-s) s|\left|f\left(s, u, u^{\prime}\right)\right| d s+\left|\frac{\lambda}{1-\alpha \eta}\right| \\
& \leq \max _{\left(s, v_{1}, v_{2}\right) \in[0,1] \times[-\beta, \beta] \times[-\beta, \beta]} \frac{1+\alpha(-\eta+1)}{1-\alpha \eta}\left|f\left(s, v_{1}, v_{2}\right)\right| \int_{0}^{1}(1-s) s d s+\left|\frac{\lambda}{1-\alpha \eta}\right| \\
& \leq \max _{\left(s, v_{1}, v_{2}\right) \in[0,1] \times[-\beta, \beta] \times[-\beta, \beta]} \frac{1+\alpha(-\eta+1)}{1-\alpha \eta} \frac{\left|f\left(s, v_{1}, v_{2}\right)\right|}{6}+\left|\frac{\lambda}{1-\alpha \eta}\right| \\
& \leq \frac{1}{1-\alpha \eta}\left[\frac{1+\alpha(1-\eta)}{6} \max \left|f\left(s, v_{1}, v_{2}\right)\right|+\lambda\right] \\
& \leq \frac{1}{1-\alpha \eta}\left[\frac{1+\alpha(1-\eta)}{6} \frac{\beta(1-\alpha \eta) 6 B}{1+\alpha(1-\eta)}+\lambda\right] \\
& \leq \frac{1}{1-\alpha \eta}[\beta(1-\alpha \eta) B+A \beta(1-\alpha \eta)] \\
& \leq \beta A+\beta B \leq \beta .
\end{aligned}
$$

Therefore, $\|u\|_{E}<\beta$ and (A2) in Theorem 1 cannot occur. Thus (A1) holds and there is $u^{*} \in E$ such that $\left\|u^{*}\right\|_{E} \leq \beta$.

Theorem 3. Suppose that $(H 1)$ holds and $f(s, u, v) \geq 0, \forall(s, u, v) \in[0,1] \times[-\beta, \beta] \times[-\beta, \beta]$. Then (1.1)-(1.2) has at least one positive solution $u^{*} \in E$.

Proof. We start the proof defining the cone $K \subset E$ by

$$
K=\left\{u \in E: u \geq 0, u(0)=0, u^{\prime}(0)=0\right\} .
$$


From $(H 1)$ and the definition of $G$ and $G_{1}$, we have that $T$ applies $K$ in $K$. As seen in the last result, $T$ is completely continuous.

We shall apply Theorem 2 . Thus, we will define $\Omega_{1}=\left\{u \in E ;\|u\|_{E}<\beta\right\}, \Omega_{2}=\left\{u \in E ;\|u\|_{E}<\right.$ $\alpha\}$ and we will show that the following conditions are true for all $u \in K$ :

(a) if $\|u\|_{E}=\alpha$ then $\|T u\|_{E} \leq \alpha$;

(b) if $\|u\|_{E}=\beta$ then $\|T u\|_{E} \geq \beta$.

In fact, the demonstration of (a) is similar to the proof of the Lemma 2. To prove (b) is necessary to verify that there is $\bar{\gamma}>0$ with

$$
\|T u\|_{E} \geq\|u\|_{E}, \quad \forall u \in K \cap \partial \Omega_{3}
$$

where $\Omega_{3}=\left\{u \in E ;\|u\|_{E}<\bar{\gamma}\right\}$.

Let us assume that the inequality is false, that is, for every $\bar{\gamma}$ such that $\beta>\bar{\gamma}>0$ there exists $u \in E$ with $\|u\|_{E}=\bar{\gamma}$ and $\|T u\|_{E}<\bar{\gamma}$. Thus for all $n \in\{1,2, \cdots\}$ with $\frac{1}{n}<\alpha$, we can find $u_{n} \in K$ such that

$$
\left\|u_{n}\right\|_{E}=\frac{1}{n} \text { and }\left\|T u_{n}\right\|_{E}<\frac{1}{n} .
$$

Then $\left\|u_{n}\right\| \longrightarrow 0$ and $\left\|T u_{n}\right\| \longrightarrow 0$, when $n \longrightarrow \infty$. Being $T$ continuous, we have $\|T 0\|_{E}=0$. On the other hand, using $(H 1)$ and the definition of $G$ and $G_{1}$ we have

$$
\begin{aligned}
\|T 0\|_{\infty} & \geq \max _{t \in[0,1]}\left\{\frac{\lambda t^{2}}{2(1-\alpha n)}\right\}, \\
& \geq \frac{\lambda}{2(1-\alpha n)}>0
\end{aligned}
$$

which is a contradiction. Therefore we have the result.

Remark 4. Note that the most important step in the proof of Theorem 3 is to impose conditions to conclude that 0 is not fixed point of $T$.

Example 3.1. Let us consider (1.1)-(1.2) with

$$
\begin{aligned}
f(t, u, v) & =\frac{1}{4} t+u^{2}+v^{2} \\
\eta & =\frac{1}{10}, \alpha=\frac{1}{3}, \lambda=\frac{1}{4}
\end{aligned}
$$

Choosing the constants

$$
\beta=10, A=0.54, B=0.45,
$$

we can easily verify that in these conditions the hypotheses (H1) are satisfied. 
Example 3.2. Let us define

$$
\begin{aligned}
f(t, u, v) & =\frac{1}{4} t+\sin (u)+\frac{1}{4} \cos (v) \\
\eta & =\frac{1}{9}, \alpha=\frac{1}{6}, \lambda \frac{14}{10}
\end{aligned}
$$

As before, choosing the constants

$$
\beta=2, A=0.75, B=0.2,
$$

we can verify that $(H 1)$ is satisfied.

\section{NUMERICAL SOLUTIONS}

In this section we show the existence and uniqueness for (1.1)-(1.2) using Banach Fixed Point Theorem. This approach is classical but very important to define numerical methods for our problem. Let us consider the iterative sequence

$$
u^{k+1}=T\left(u^{k}\right)
$$

and the basic assumptions

$$
\begin{aligned}
& \text { (H2) }\left|f\left(s, u, u^{\prime}\right)-f\left(s, v, v^{\prime}\right)\right| \leq A \max \left\{|u(s)-v(s)|,\left|u^{\prime}(s)-v^{\prime}(s)\right|\right\} \\
& \text { (H3) } \frac{-t^{2}+t}{2}+\frac{\alpha t \eta(-\eta+1)}{2(1-\alpha \eta)} \leq \frac{1}{A} .
\end{aligned}
$$

Theorem 1. Suppose that (H1), (H2) and (H3) are satisfied. Then (1.1)- (1.2) has a unique solution $u$ with $\|u\|_{E} \leq \beta$. Moreover, $u^{k+1}=T\left(u^{k}\right) \rightarrow u$.

Proof. Let us consider $u, v \in \Omega$ with $\|u\|_{E} \leq \beta$ and $\|v\|_{E} \leq \beta$. Then

$$
\begin{aligned}
\| T u- & T v\left\|_{E}=\right\|(T u-T v)^{\prime} \|_{\infty} \\
& =\left|\int_{0}^{1} G_{1}(t, s)\left[f\left(s, u, u^{\prime}\right)-f\left(s, v, v^{\prime}\right)\right] d s+\frac{\alpha t}{1-\alpha \eta} \int_{0}^{1} G_{1}(t, s)\left[f\left(s, u, u^{\prime}\right)-f\left(s, v, v^{\prime}\right)\right] d s\right| \\
& \leq A \max _{s}\left\{|u(s)-v(s)|,\left|u^{\prime}(s)-v^{\prime}(s)\right|\right\}\left(\int_{0}^{1} G_{1}(t, s) d s+\frac{\alpha t}{1-\alpha \eta} \int_{0}^{1} G_{1}(\eta, s) d s\right) \\
& \leq A \max _{s}\left\{|u(s)-v(s)|,\left|u^{\prime}(s)-v^{\prime}(s)\right|\right\}\left(\frac{-t^{2}+t}{2}+\frac{\alpha t \eta(-\eta+1)}{2(1-\alpha \eta)}\right)
\end{aligned}
$$

Using (H3) we obtain

$$
\begin{aligned}
& \leq A \max _{s}\left\{|u(s)-v(s)|,\left|u^{\prime}(s)-v^{\prime}(s)\right|\right\} \frac{1}{A} \\
& \leq \max _{s}\left\{|u(s)-v(s)|,\left|u^{\prime}(s)-v^{\prime}(s)\right|\right\}=\|u-v\|_{E}
\end{aligned}
$$

Motivated by the last result we can define Algorithm 1.

In sequence we are presenting some examples in order to establish the effectiveness of Algorithm 1. In tables, $\varepsilon_{u}^{k}$ denotes $\left\|u^{*}-u^{k}\right\|_{\infty}$ where $u^{*}$ is the exact solution, $\varepsilon^{k}$ denotes $\left\|u^{k+1}-u^{k}\right\|_{\infty}$ and $\bar{\varepsilon}^{k}=\frac{\left\|u^{k+1}-u^{k}\right\|_{\infty}}{\left\|u^{k+1}\right\|_{\infty}}$. Still, "It" denotes "iteration". 


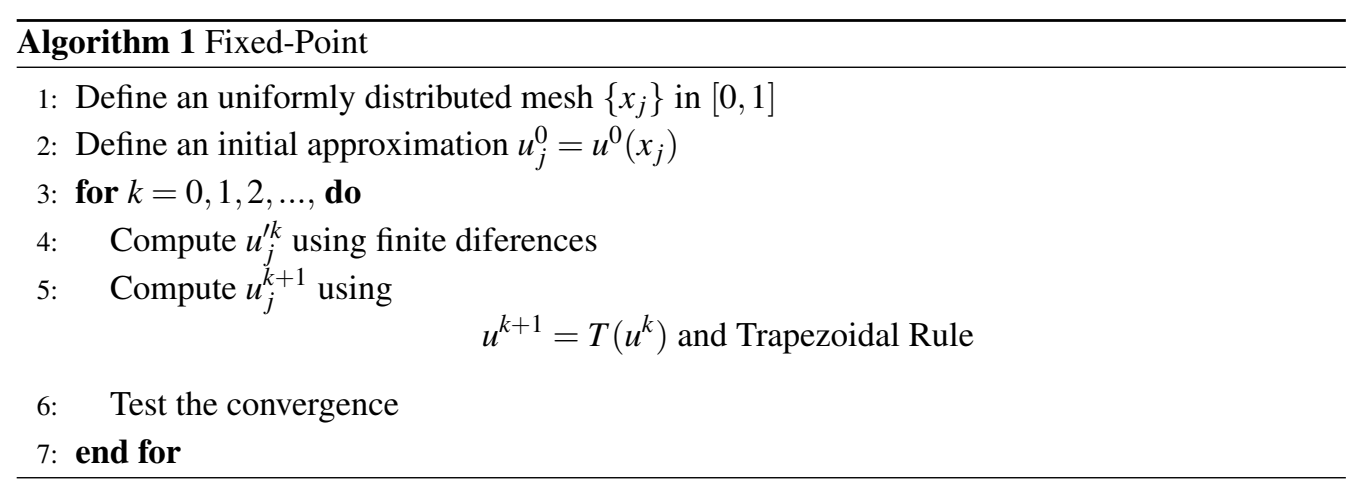

Example 4.1. In this example, we consider

$$
\begin{aligned}
f\left(x, u, u^{\prime}\right) & =-u^{\prime} \\
\eta & =\frac{\pi}{4}, \alpha=\frac{1}{10}, \lambda=0.770760306689242
\end{aligned}
$$

The the analytical solution is $u^{*}(x)=1-\cos (x)$. The Table 1 contains results of application in Example 4.1.

We can make additional tests. From Theorem 3 we have a solution for Examples 3.1 and 3.2 but in both case, we do not know which they are. Let us apply Algorithm 1 in these problems. For this purpose, we can consider the condition

$$
\frac{\left\|u^{k+1}-u^{k}\right\|}{\left\|u^{k+1}\right\|_{\infty}}<10^{-4}
$$

as stopping criterion for the algorithm. The results for these examples are presented in Table 2 and 3, respectively. The illustrations of these results are given in Figure 1 and 2. 
Table 1: Algorithm 1 considering Example 4.1.

\begin{tabular}{|c|c|c|c|}
\hline It & $\varepsilon_{u}^{k}$ & $\varepsilon^{k}$ & $\bar{\varepsilon}^{k}$ \\
\hline 1 & 0.104585227251908 & 0.355112466879952 & 1.000000000000000 \\
2 & 0.072538564385106 & 0.032046662866802 & 0.082773878760819 \\
3 & 0.069264937033799 & 0.003273627351307 & 0.008384612437847 \\
4 & 0.068925441261629 & 0.000339495772170 & 0.000868781674432 \\
5 & 0.068890166416009 & 0.000035274845620 & 0.000090261428243 \\
\hline
\end{tabular}

Table 2: Algorithm 1 considering Example 3.1.

\begin{tabular}{|c|c|c|c|}
\hline It & $\varepsilon_{u}^{k}$ & $\varepsilon^{k}$ & $\bar{\varepsilon}^{k}$ \\
\hline 1 & - & 0.168278823890335 & 1.000000000000000 \\
2 & - & 0.007563461402919 & 0.043012756518181 \\
3 & - & 0.000744660869474 & 0.004216964422657 \\
4 & - & 0.000077049209989 & 0.000436134198292 \\
\hline
\end{tabular}

Table 3: Algorithm 1 considering Example 3.2.

\begin{tabular}{|c|c|c|c|}
\hline It & $\varepsilon_{u}^{k}$ & $\varepsilon^{k}$ & $\bar{\varepsilon}^{k}$ \\
\hline 1 & - & 0.740971458506793 & 1.000000000000000 \\
2 & - & 0.010141509530254 & 0.013876702158219 \\
3 & - & 0.000276799190473 & 0.000378602975785 \\
4 & - & 0.000007307338952 & 0.000009995000056 \\
\hline
\end{tabular}

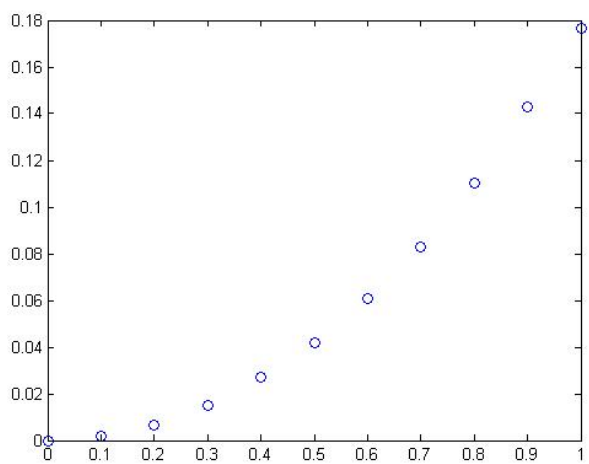

Figure 1: Numerical solution obtained from Example 1 using Algorithm 1.

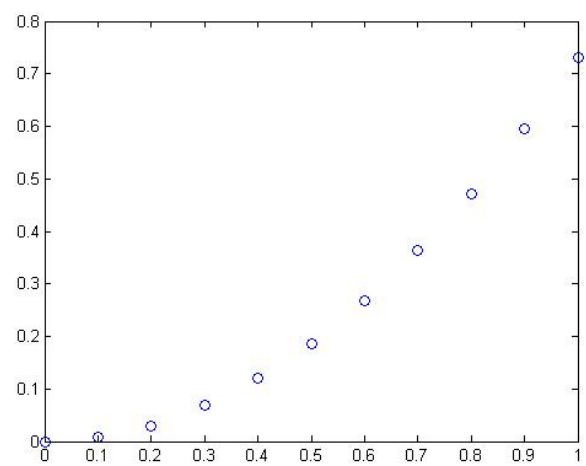

Figure 2: Numerical solution obtained from Example 2 using Algorithm 1. 
RESUMO. Neste artigo, consideramos uma equação com três pontos de fronteira de terceira ordem com condições de contorno não homogêneas. Com uso do Teorema de Krasnoselskii e da Alternativa de Leray-Schauder, apresentamos resultados de existência para soluções positivas. Exemplos não triviais são fornecidos e um método numérico é introduzido.

Palavras-chave: soluções numéricas, terceira-ordem, problema de valor de contorno, Teorema de Krasnoselskii.

\section{REFERENCES}

[1] R.P. Agarwal, M. Meehan \& D. O'Regan. "Fixed point theory and applications", volume 141. Cambridge university press (2001).

[2] D. Anderson. Multiple Positive Solutions for a Three-Point Boundary Value Problem. Mathematical and Computer Modelling, 27(6) (1998), 49-57.

[3] D.R. Anderson. Green's function for a third-order generalized right focal problem. Journal of Mathematical Analysis and Applications, 288(1) (2003), 1-14. doi:10.1016/S0022-247X(03) 00132-X.

[4] D.R. Anderson \& J.M. Davis. Multiple Solutions and Eigenvalues for Third-Order Right Focal Boundary Value Problems. Journal of Mathematical Analysis and Applications, 267(1) (2002), 135157. doi:10.1006/jmaa.2001.7756. URL http://linkinghub.elsevier.com/retrieve/pii/ S0022247X0197756X.

[5] A. Boucherif \& N. Al-Malki. Nonlinear three-point third-order boundary value problems. Applied Mathematics and Computation, 190(2) (2007), 1168-1177. doi:10.1016/j.amc.2007.02.039. URL http://linkinghub.elsevier.com/retrieve/pii/S0096300307001476.

[6] H. Chen. Positive solutions for the nonhomogeneous three-point boundary value problem of secondorder differential equations. Math. Comput. Modelling, 45(7-8) (2007), 844-852. doi:10.1016/j.mcm. 2006.08.004. URL http://dx.doi.org/10.1016/j.mcm.2006.08.004.

[7] Z.B. Fei \& Xiangli. Existence of triple positive solutions for a third order generalized right focal problem. Math. Inequal. Appl, 9(3) (2006), 2006.

[8] J. Graef \& B. Yang. Multiple Positive Solutions to a Three Point Third Order Boundary Value Problem. Discrete and Continuous Dynamical Systems, 2005 (2005), 337-344.

[9] Y. Lin \& M. Cui. A numerical solution to nonlinear multi-point boundary value problems in the reproducing kernel space. Mathematical Methods in the Applied Sciences, 34(1) (2011), 44-47. doi: 10.1002/mma.1327.

[10] Z. Liu \& F. Li. On the existence of positive solutions of an elliptic boundary value problem. Chinese Ann. Math. Ser. B, 21(4) (2000), 499-510. doi:10.1142/S0252959900000492. URL http://dx . doi . org/10.1142/S0252959900000492. 
[11] R. Ma. Existence Theorems for a Second Order m-Point Boundary Value Problem. Journal of Mathematical Analysis and Applications, 211(2) (1997), 545-555. doi:10.1006/jmaa.1997.5416. URL http://www.sciencedirect.com/science/article/pii/S0022247X97954160.

[12] Y.H. Ma \& R.Y. Ma. Positive solutions of a singular nonlinear three-point boundary value problem. Acta Math. Sci. Ser. A Chin. Ed., 23(5) (2003), 583-588. doi:10.1016/j.jmaa.2004.10.029.

[13] Y. Sun. Positive solutions for third-order three-point nonhomogeneous boundary value problems. Applied Mathematics Letters, 22(1) (2009), 45-51. doi:10.1016/j.aml.2008.02.002. URL http:// linkinghub.elsevier.com/retrieve/pii/S0893965908000827.

[14] Q.L. Yao. The existence and multiplicity of positive solutions for a third-order three-point boundary value problem. Acta Mathematicae Applicatae Sinica, 19(1) (2003), 117-122. doi:10.1007/ s10255-003-0087-1.

[15] H. Yu, L. Haiyan \& Y. Liu. Multiple positive solutions to third-order three-point singular semipositone boundary value problem. Proceedings Mathematical Sciences, 114(4) (2004), 409-422. 